\title{
$\widehat{A}$ Madridge \\ madridge Journal of Otorhinolaryngology \\ interconnecting Scientific World
}

Case Report

Open Access

\section{Sinusitis with Extensive Neck, Orbit \& Scalp Involvement - A Rare Presentation}

\author{
Anil S Harugop ${ }^{1}$, Shama A Bellad ${ }^{2}$, Deepa Sethunath ${ }^{3}$, Rajesh R Havaldar ${ }^{3 \star}$ and Deepika Reddy \\ ${ }^{1}$ Professor and Head, Department of ENT and HNS, J.N.Medical College, Belagavi, Karnataka, India \\ ${ }^{2}$ Assistant Professor, Department of ENT and HNS, J.N.Medical College, Belagavi, Karnataka, India \\ ${ }^{3}$ Post Graduate Resident, Department of ENT and HNS, J.N.Medical College, Belagavi, Karnataka, India
}

\section{Article Info}

\author{
*Corresponding author: \\ Rajesh R Havaldar \\ Department of E.N.T and H.N.S \\ J.N.Medical College \\ Kaher, Belagavi \\ India \\ Tel: 7090095006 \\ E-mail: rajeshhavaldar@yahoo.com
}

Received: August 13, 2018
Accepted: October 4, 2018
Published: October 12, 2018

Citation: Harugop AS, Bellad SA, Sethunath D, Havaldar RR, Reddy D. Sinusitis with Extensive Neck, Orbit \& Scalp Involvement - A Rare Presentation. Madridge J Otorhinolaryngol. 2018; 4(1): 70-72.

doi: $10.18689 / \mathrm{mjol}-1000114$

Copyright: @ 2018 The Author(s). This work is licensed under a Creative Commons Attribution 4.0 International License, which permits unrestricted use, distribution, and reproduction in any medium, provided the original work is properly cited.

Published by Madridge Publishers

\begin{abstract}
Sinusitis complicating to involve the eye, neck and scalp is a rare but theoretically possible complication. We had a case of a 60 -year-old female patient who was immuno competent and developed neck, orbit and scalp abscess as a complication of sinusitis. She was effectively managed by surgical debridement and antibiotics. A high degree of suspicion is mandatory in patients with sinusitis and prompt surgical intervention in cases not responding to antibiotic therapy is of paramount importance.
\end{abstract}

Keywords: Sinusitis; Neck, Orbit and Scalp Abscess; Rhinosinusitis; Paranasal sinuses.

\section{Introduction}

A complication of rhinosinusitis may be defined as any adverse progression of chronic or acute bacterial infection beyond the paranasal sinuses, or compromise in function in any part of the body due to local or distant effects of the condition. The most important and often encountered complication of ethmoid rhinosinusitis is orbital cellulitis, which can present in any form. It is far more common in children $(50 \%$ in under 6 years) and young adults (76-85\% under 20 years) [1]. Necrotizing fasciitis of facial region is even a rare occurrence, especially in an immunocompetent patient. Cervicofacial necrotizing fasciitis is most commonly as a complication of dental disease [2]. Maxillary sinusitis is a rare cause of necrotizing fasciitis. Clinically, the onset is commonly insidious in the form of nonspecific regional facial swelling, erythema, and fever. Hence, clinical differentiation from more benign pathologies of the neck, such as cellulitis, may be impossible at an early stage. Without prompt surgical treatment, necrotizing fasciitis of the head and neck would often lead to mediastinitis and fatal sepsis [3]. Therefore there needs to be no delay in aggressively managing such cases in order to prevent fatal outcomes.

\section{Case Report}

60 year old female presented with swelling of the left eye since 3 days insidious in onset, gradually progressive, initially the patient was able to open her eye with reduced eye movements and gradually the swelling progressed (Figure 1). She had generalized weakness and was dehydrated on presentation. 


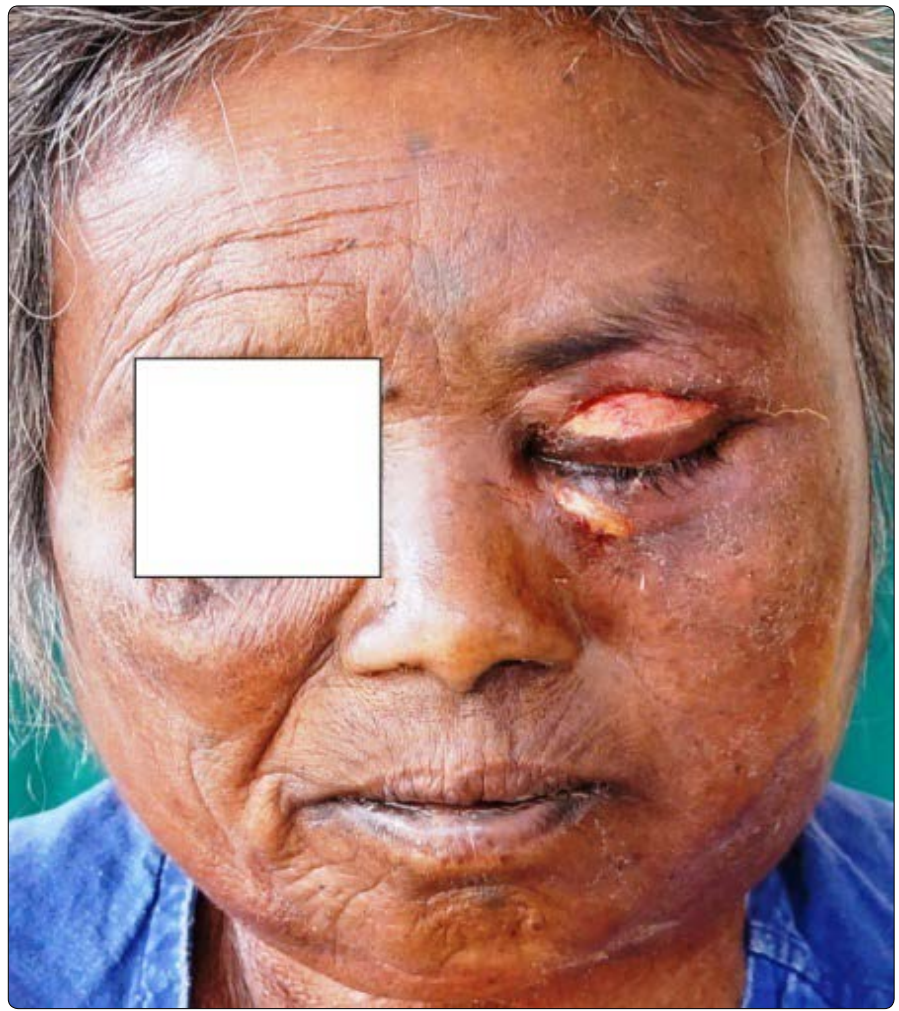

Figure 1. Showing patient at presentation.

Hence, the vision could not be assessed. There was pain in the left eye pricking type, radiating to the left side of the face, left ear, and to the neck. This was associated with purulent discharge from the left upper and lower eyelid since 1 day which was foul smelling and blood tinged. The patient had no history of redness/ watering of the eye. There was no history of trauma to the eyes, no history of previous surgeries to the eye/nose. History of recurrent episodes of nasal discharge, mucoid, non foul smelling, non blood tinged since one month. No history of Diabetes Mellitus, any drug reaction or insect bite. She is a known hypertensive on oral hypertensive medication for the last 10 years. She gave a history of tobacco chewing. Her blood urea, serum creatinine and sugar levels were within normal limits. There was an elevated total leukocyte count with neutrophilic leucocytosis in the peripheral blood smear examination.

Examination revealed Grade 2 proptosis of the left eye with pus discharging from the left upper eyelid. ENT Examination showed a diffuse firm, non fluctuant, tender swelling involving the left side of the face extending from midline of the face to the left cheek with local rise of temperature. An ulcer was seen over the lateral aspect of the left upper eyelid, $7 \times 3 \mathrm{~mm}$ with a discharging sinus in its medial end. The discharge was thick, yellowish, purulent and foul-smelling. Nose examination on Anterior Rhinoscopy showed foul smelling discharge in the left nasal cavity, left side pan sinus tenderness.

CT PNS (Figure 2) showed left maxillary, ethmoidal and frontal sinusitis with bony erosions of left lamina papyracea and mottling suggestive of a probable fungal etiology. There was left orbital and subcutaneous cellulitis. Patient was started on antibiotics covering Gram positive and Gram negative organisms.

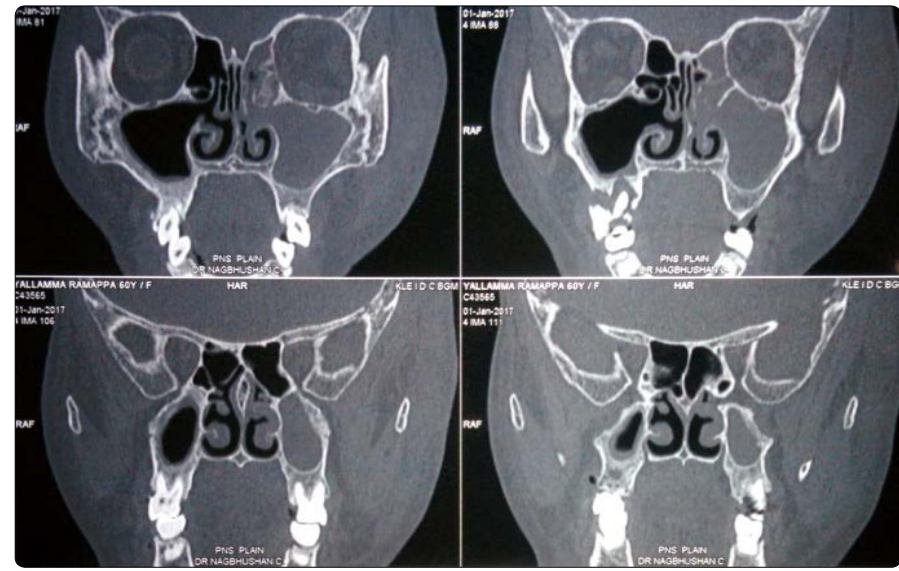

Figure 2. CT PNS showing mottling due to fungal sinusitis in left maxilla with breach in lamina papyracea (arrow head).

She was taken up for Immediate Functional Endoscopic Sinus Surgery with Left Orbital Decompression. Left orbital decompression was done and at the apex of the orbit through the Lamina Papyracea about $5 \mathrm{ml}$ of pus was aspirated Stankiewicz sign (Figure 3). The pus was sent for culture and sensitivity. In addition, $\mathrm{KOH}$ test for fungal elements was negative.

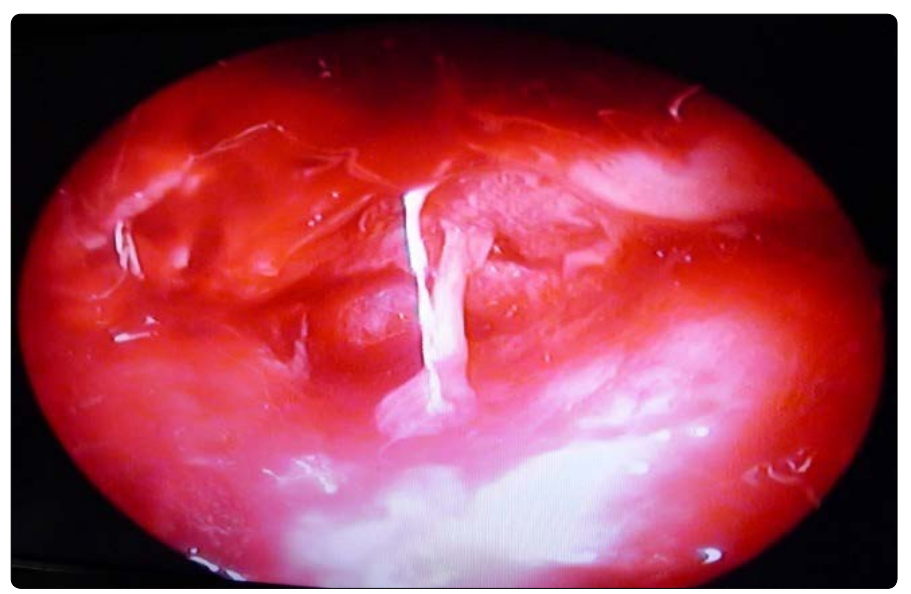

Figure 3. Demonstrating Stankiewicz sig

On postoperative day 2 , we noticed an increase in the size of the left facial swelling with spontaneous rupture at the left supraorbital and infraorbital region. Incision and Drainage of left facial cellulitis was done using oblique incision $2 \mathrm{~cm}$ long about $2 \mathrm{~cm}$ above the midline between angle of mandible and symphysis menti (Figure 4).

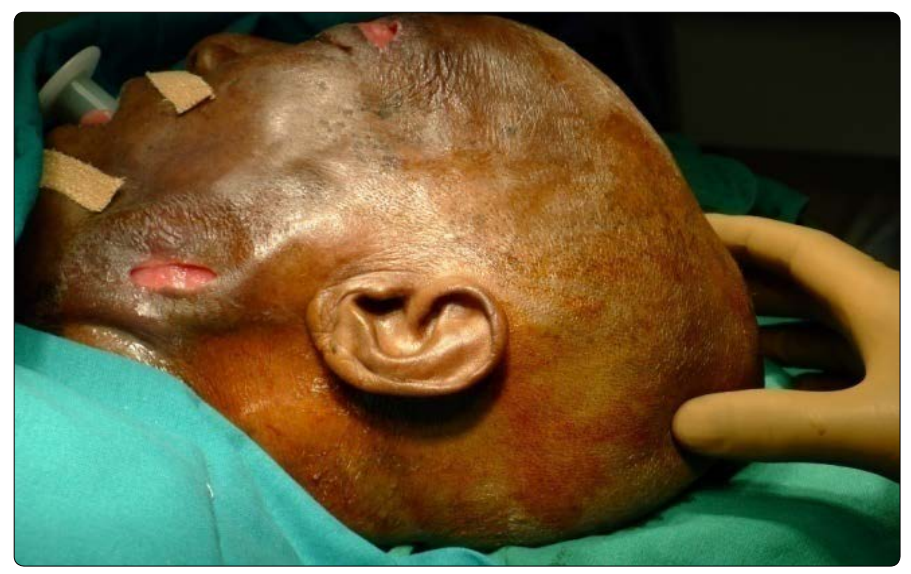

Figure 4. Left facial cellulitis - incision and drainage 
She became disoriented and a physician opinion was taken and then she was diagnosed as Metabolic Encephalopathy secondary to elevated serum creatinine and electrolyte imbalance. She was started on higher antibiotics covering anaerobic organisms as well. Culture and sensitivity samples resulted in a positive identification with Acinetobacter $s p$.

On Postoperative Day 9, the patient developed scalp cellulitis with abscess and was started on Linezolid (Figure 5).

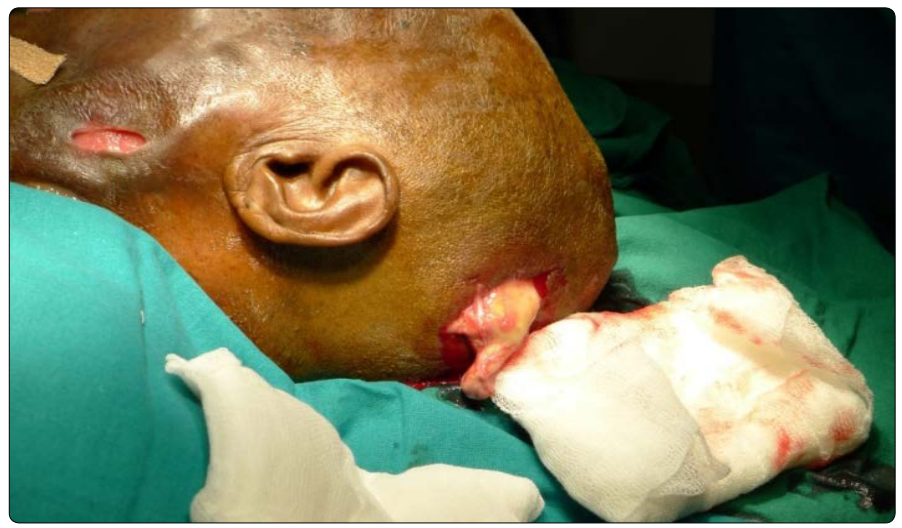

Figure 5. Scalp abscess incision and drainage

On Postoperative Day 10, Incision and drainage of scalp abscess was done and 12 days later secondary suturing was done. After 10 days of secondary suturing the patient improved symptomatically, the proptosis reduced and eyeball movements improved. The swelling in the scalp reduced and there was also an overall improvement in the general condition of the patient and the patient was discharged (Figure 6).

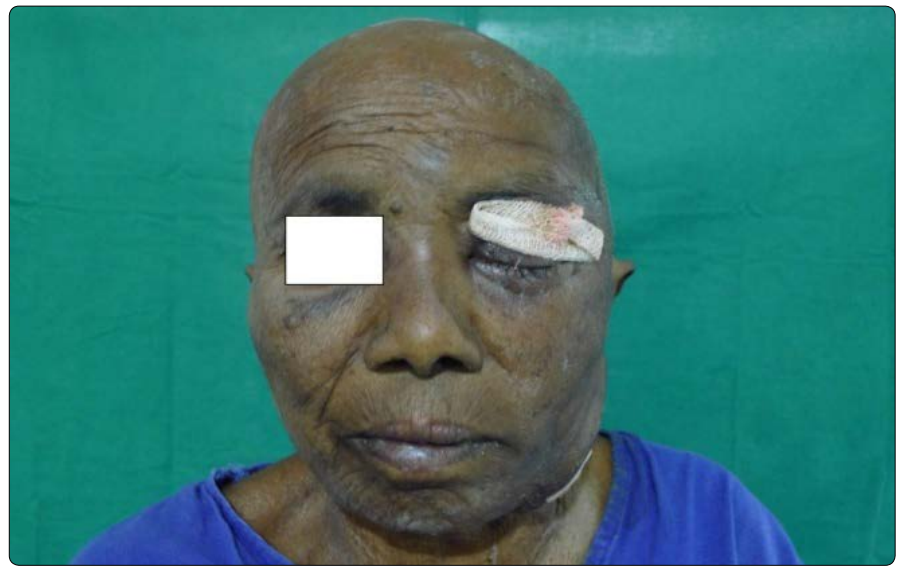

Figure 6. Patient before discharge

\section{Discussion}

Younis et al (2002), in their study mentioned that an infection from sinuses can easily spread to the orbit and to the intracranial cavity as these anatomical structures are very closely interrelated. The most common complication of sinusitis is orbital cellulitis followed by intracranial complications like meningitis, brain abscess and cavernous sinus thrombosis. Other complications include mucocele, pyelocele, osteomyelitis, facial cellulitis and subperiosteal abscess [4].

In a 15 year review of 90 patients with orbital cellulitis complicating sinusitis done by Nwaorgu (2004), sinuses were the origin of the infection in 57\% patients [5].

In a study of 218 patients of orbital cellulitis, done by
Choudhary et al. (2007), it was found that sinusitis was the most common predisposing factor [6].

Mills RP and Kartush JM (1985) concluded in their study that ethmoidal, maxillary or frontal sinusitis could complicate into orbital subperiosteal abscess. Direct extension through the sinus wall appeared to be more commonly involved than the rarer route of local thrombophlebitis [7].

According to Nugent et al (2010), in the pre-antibiotic era, infection used to extend to the frontal bone as an extension from the frontal sinus commonly [8]. This is known as'Pott's puffy tumors' which is a subperiosteal abscess formed from a pre existing frontal bone osteomyelitis [9].

Although there are many established routes of spread for diseases involving the paranasal sinuses to the orbit [10], there was no literature evidence suggestive of infection spread to the neck, face and scalp. However, spread of cellulitis via the subcutaneous route can be a possible route of spread in such cases.

In our case we isolated the bacterium Acinetobacter species in one of the culture samples. This is consistent with the study done by Nugent et al. [8]. But reporting a coincident neck abscess with orbital cellulitis along with a scalp abscess as a complication of sinusitis is a rare but possible finding.

\section{Conclusion}

The complications of acute rhino sinusitis have the dangerous potential to be life threatening. A high degree of suspicion and a continuous watch is needed on patients with sinusitis not improving with standard antibiotic therapy.

Early patient identification, appropriate medical therapy for adequate period as advocated and emergency surgical intervention when in need by ophthalmologists, ENT surgeons and neurosurgeons offer a favorable outcome.

\section{Conflicts of Interest}

The Authors declare that there is no conflict of interest

\section{References}

1. Slack R, Sim R. Complications of rhinosinusitis. Scott-Brown's Otorhinolaryngology, Head and Neck Surgery. 2008; 120: 1539-1548.

2. Raboso E, Llavero MT, Rosell A, Martinez-Vidal A. Craniofacial necrotizing fasciitis secondary to sinusitis. J Laryngol Otol. 1998; 112(4): 371-2. doi: 10.1017/S0022215100140496

3. Gupta N, Varshney S, Gupta P. Facial Necrotizing Fasciitis: A Rare Complication of Maxillary Sinusitis. Indian J Clin Pract. 2013; 24(7): 628-630.

4. Younis RT, Lazar RH, Bustillo A, Anand VK. Orbital infection as a complication of sinusitis: are diagnostic and treatment trends changing. Ear Nose Throat J. 2002; 8(11): 771-775.

5. Nwaorgu OGB, Awobem FJ, Onakoya PA, Wabem AAA. Orbital cellulitis complicating sinusitis: a 15 year review. Niger J Surg Res. 2004; 6(1-2): 14 16. doi: 10.4314/njsr.v6i1-2.54769

6. Chaudhry IA, Shamsi FA, Elzaridi E, et al. Outcome of treated orbital cellulitis in a tertiary eye care centre in the middle East. Ophthalmology. 2007; 114(2): 345-354.

7. Mills RP, Kartush JM. Orbital wall thickness and the spread of infection from the paranasal sinuses. Clin Otolaryngol Allied Sci. 1985; 10(4): 209-216.

8. Nugent NF, Murphy $M$, Kelly J. Scalp abscess-a cautionary tale. J Plast Reconstr Aesthet Surg. 2010; 63(8): e619-e621. doi: 10.1016/j.bjps.2010.02.011

9. Flamm ES. Percivall Pott: an 18th century neurosurgeon. J Neurosurg. 1992; 76(2): 319-326.

10. Gleeson M, Browning GG, Burton MJ. Scott-Brown's Otorhinolaryngology, Head and Neck Surgery. $7^{\text {th }}$ Edn. Ann R Coll Surg Engl. 2011; 93(7): 559. doi: 10.1308/147870811X598605b 